\title{
NLMS Is More Robust to Input-Correlation Than LMS: A Proof
}

\author{
Anum Ali, Member, IEEE, Muhammad Moinuddin, Member, IEEE, \\ and Tareq Y. Al-Naffouri, Senior Member, IEEE
}

\begin{abstract}
In this work, we comparatively analyze the least mean squares (LMS) algorithm and the normalized least mean squares (NLMS) algorithm. We use the input moment matrices for comparison as the mean-square behavior of both algorithms is determined by the input moment matrices. First, we derive the closed-form expressions of the input moment matrices of the NLMS. Second, we do a numerical and theoretical comparison of the input moment matrices of the LMS and the NLMS. The analysis shows why the performance of the NLMS is less sensitive to the changes in eigenvalue-spread (of the input-correlation matrix) than the LMS.
\end{abstract}

Index Terms-Adaptive filters, LMS, NLMS, input moment matrices, eigenvalue-spread.

\section{INTRODUCTION}

Adaptive filters find applications in diverse fields of signal processing, e.g., equalization, noise cancellation, linear prediction, and system identification [1]-[3]. The least mean squares (LMS) [4] is the simplest and most commonly used adaptive algorithm. The convergence properties of the LMS, however, depend on input-correlation and it converges slowly for highly correlated signals like speech. Its "normalized" version, i.e., normalized least mean squares (NLMS) [5], is less sensitive to input-correlation due to input normalization. Although there have been several attempts to explain when and why the NLMS can perform better than the LMS [1], [3], [6]-[18], no precise mathematical justification is provided. This work aims to justify why NLMS is more robust to the input-correlation than LMS. We do so by exploiting the fact that the mean-square performance of both the algorithms (i.e., LMS and NLMS) is characterized by input moment matrices - given further ahead in (4) and (5). Specifically, we take two approaches

- An empirical approach: we compare the sensitivity of the entries of the two sets of input moment matrices evaluated in closed-form - to the random perturbation in the eigenvalues of the input-correlation matrix.

(Corresponding author: T. Y. Al-Naffouri)

A. Ali is with the Standards and Mobility Innovation Laboratory, Samsung Research America, Plano, TX 75023, USA (e-mail: anum.ali@ samsung.com).

M. Moinuddin is with the Department of Electrical and Computer Engineering, King Abdulaziz University, Jeddah 21589, Saudi Arabia, and also with the Center of Excellence in Intelligent Engineering Systems (CEIES), King Abdulaziz University, Jeddah 21589, Saudi Arabia (e-mail: mmsansari@ kau.edu.sa).

T. Y. Al-Naffouri is with the Computer, Electrical and Mathematical Sciences and Engineering Division, King Abdullah University of Science and Technology, Thuwal 23955-6900, Saudi Arabia (e-mail: tareq.alnaffouri@kaust.edu.sa).
- A theoretical approach: we analyze the change in the range of the entries of the two sets of input moment matrices with the eigenvalue-spread of the input-correlation matrix.

We start with a brief overview of the two algorithms. At each time instant $i$, the objective of an adaptive filter is to generate the weight vector $\mathbf{w}_{i} \in \mathbb{C}^{M}$ using input regressor $\mathbf{u}_{i} \in \mathbb{C}^{M}$, such that $\mathbf{u}_{i}^{T} \mathbf{w}_{i}$ (where superscript $T$ is the transpose) can be an estimate of some desired response $d_{i}$. For identifying an unknown system $\mathbf{w}^{o} \in \mathbb{C}^{M}$, the desired response $d_{i}$ can be expressed as

$$
d_{i}=\mathbf{u}_{i}^{T} \mathbf{w}^{o}+v_{i}
$$

where $v_{i} \sim \mathcal{C N}\left(0, \sigma_{v}^{2}\right)$ is the additive noise. Consider an adaptive filter that seeks to minimize the estimation error $e_{i}=d_{i}-\mathbf{u}_{i}^{T} \mathbf{w}_{i-1}$. Specifically, the weight update recursion of the filter with step-size $\alpha$ has the form

$$
\mathbf{w}_{i}=\mathbf{w}_{i-1}+\alpha \frac{\mathbf{u}_{i}^{\mathrm{c}}}{g\left(\mathbf{u}_{i}\right)} e_{i}, i \geq 0, \mathbf{w}_{-1}=\mathbf{0},
$$

where superscript $\mathrm{c}$ is the complex conjugate, and $g\left(\mathbf{u}_{i}\right)$ is some function of the input. For the LMS $g\left(\mathbf{u}_{i}\right)=1$, and for the NLMS $g\left(\mathbf{u}_{i}\right)=\left\|\mathbf{u}_{i}\right\|^{2}$, where $\left\|\mathbf{u}_{i}\right\|^{2} \triangleq \mathbf{u}_{i}^{*} \mathbf{u}_{i}$ and superscript $*$ is Hermitian transpose. The mean squared error (MSE) of the LMS is described by the following weighted variance relation [2], [19]

$$
\mathbb{E}\left[\left\|\tilde{\mathbf{w}}_{i}\right\|_{\boldsymbol{\Sigma}}^{2}\right]=\mathbb{E}\left[\left\|\tilde{\mathbf{w}}_{i-1}\right\|_{\left(\mathbf{I}-\alpha \mathbf{A}_{\mathrm{L}}+\alpha^{2} \mathbf{B}_{\mathrm{L}}\right) \boldsymbol{\Sigma}}^{2}\right]+\alpha^{2} \sigma_{v}^{2} \operatorname{tr}\left[\mathbf{C}_{\mathrm{L}} \boldsymbol{\Sigma}\right],
$$

where $\mathbb{E}[\cdot]$ is the expectation operator, $\tilde{\mathbf{w}}_{i}=\mathbf{w}^{o}-\mathbf{w}_{i}$ is the weight error vector, $\boldsymbol{\Sigma}$ is a weighting matrix (different performance measures can be obtained by appropriately choosing $\boldsymbol{\Sigma}$ ), and $\operatorname{tr}(\cdot)$ is the trace operator. Thus, the matrices $\mathbf{A}_{\mathrm{L}}$, $\mathbf{B}_{\mathrm{L}}$, and, $\mathbf{C}_{\mathrm{L}}$ defined in (4) completely characterize the meansquare behavior of the LMS

$$
\begin{aligned}
& \mathbf{A}_{\mathrm{L}} \triangleq 2 \mathbb{E}\left[\mathbf{u}_{i} \mathbf{u}_{i}^{*}\right]=2 \boldsymbol{\Lambda}, \\
& \mathbf{B}_{\mathrm{L}} \triangleq \mathbb{E}\left[\left(\mathbf{u}_{i} \mathbf{u}_{i}^{*}\right)^{T} \odot\left(\mathbf{u}_{i} \mathbf{u}_{i}^{*}\right)\right]=2 \boldsymbol{\Lambda}^{2}+\boldsymbol{\lambda} \boldsymbol{\lambda}^{T}, \text { and } \\
& \mathbf{C}_{\mathrm{L}} \triangleq \mathbb{E}\left[\mathbf{u}_{i} \mathbf{u}_{i}^{*}\right]=\boldsymbol{\Lambda},
\end{aligned}
$$

where $\mathbf{u}_{i} \sim \mathcal{C N}(\mathbf{0}, \boldsymbol{\Lambda})$, and $\boldsymbol{\Lambda}$ - the input-correlation matrix - can be assumed diagonal for the sake of mean-square analysis without loss of generality [20], [21]. Further, $\odot$ is the Hadamard product, and $\boldsymbol{\lambda}=\operatorname{diag}(\boldsymbol{\Lambda})=\left[\lambda_{1}, \lambda_{2}, \cdots, \lambda_{M}\right]^{T}$ is the vector of the eigenvalues of input-correlation matrix. Similarly, the "normalized" versions of the LMS moment matrices defined in (5) completely characterize the mean- 
square behavior of the NLMS. The reason is that the same weighted variance relation (3) applies to the NLMS simply by replacing $\mathbf{A}_{\mathrm{L}}, \mathbf{B}_{\mathrm{L}}$, and, $\mathbf{C}_{\mathrm{L}}$, by the normalized counterparts $\mathbf{A}_{\mathrm{N}}, \mathbf{B}_{\mathrm{N}}$, and, $\mathbf{C}_{\mathrm{N}}$. These normalized moment matrices are

$$
\begin{aligned}
& \mathbf{A}_{\mathrm{N}} \triangleq 2 \mathbb{E}\left[\frac{\mathbf{u}_{i} \mathbf{u}_{i}^{*}}{\left\|\mathbf{u}_{i}\right\|^{2}}\right], \\
& \mathbf{B}_{\mathrm{N}} \triangleq \mathbb{E}\left[\frac{\left(\mathbf{u}_{i} \mathbf{u}_{i}^{*}\right)^{T} \odot\left(\mathbf{u}_{i} \mathbf{u}_{i}^{*}\right)}{\left(\left\|\mathbf{u}_{i}\right\|^{2}\right)^{2}}\right], \text { and } \\
& \mathbf{C}_{\mathrm{N}} \triangleq \mathbb{E}\left[\frac{\mathbf{u}_{i} \mathbf{u}_{i}^{*}}{\left(\left\|\mathbf{u}_{i}\right\|^{2}\right)^{2}}\right] .
\end{aligned}
$$

While the input moment matrices of the LMS are easily expressed in closed-form, evaluation of the moment matrices of the NLMS has remained elusive. In the next section, we express these moments in closed-form.

\section{Closed-Form expressions of $\mathbf{A}_{\mathrm{N}}, \mathbf{B}_{\mathrm{N}}$, AND $\mathbf{C}_{\mathrm{N}}$}

To evaluate the NLMS moment matrices, rewrite them in factored form as follows

$$
\mathbf{A}_{\mathrm{N}}=\boldsymbol{\Lambda} \overline{\mathbf{A}}_{\mathrm{N}}, \quad \mathbf{B}_{\mathrm{N}}=\boldsymbol{\Lambda} \overline{\mathbf{B}}_{\mathrm{N}} \boldsymbol{\Lambda}, \quad \text { and } \quad \mathbf{C}_{\mathrm{N}}=\boldsymbol{\Lambda} \overline{\mathbf{C}}_{\mathrm{N}},
$$

where $\overline{\mathbf{A}}_{\mathrm{N}}, \overline{\mathbf{B}}_{\mathrm{N}}$, and $\overline{\mathbf{C}}_{\mathrm{N}}$ are the "whitened" versions of $\mathbf{A}_{\mathrm{N}}$, $\mathbf{B}_{\mathrm{N}}$, and $\mathbf{C}_{\mathrm{N}}$, respectively. Specifically, let $\overline{\mathbf{u}}_{i} \sim \mathcal{C N}(\mathbf{0}, \mathbf{I})$ such that $\mathbf{u}_{i}=\boldsymbol{\Lambda}^{1 / 2} \overline{\mathbf{u}}_{i}$, then

$$
\begin{aligned}
& \overline{\mathbf{A}}_{\mathrm{N}} \triangleq 2 \mathbb{E}\left[\frac{\overline{\mathbf{u}}_{i} \overline{\mathbf{u}}_{i}^{*}}{\left\|\overline{\mathbf{u}}_{i}\right\|_{\Lambda}^{2}}\right], \\
& \overline{\mathbf{B}}_{\mathrm{N}} \triangleq \mathbb{E}\left[\frac{\left(\overline{\mathbf{u}}_{i} \overline{\mathbf{u}}_{i}^{*}\right)^{T} \odot\left(\overline{\mathbf{u}}_{i} \overline{\mathbf{u}}_{i}^{*}\right)}{\left(\left\|\overline{\mathbf{u}}_{i}\right\|_{\boldsymbol{\Lambda}}^{2}\right)^{2}}\right], \text { and } \\
& \overline{\mathbf{C}}_{\mathrm{N}} \triangleq \mathbb{E}\left[\frac{\overline{\mathbf{u}}_{i} \overline{\mathbf{u}}_{i}^{*}}{\left(\left\|\overline{\mathbf{u}}_{i}\right\|_{\Lambda}^{2}\right)^{2}}\right] .
\end{aligned}
$$

Let the $k$ th entry of a vector $\mathbf{x}$ be represented as $x(k)$, and define the (scalar) random variables, $s_{k} \triangleq \frac{|\bar{u}(k)|^{2}}{\|\overline{\mathbf{u}}\|_{\Lambda}^{2}}, s_{k \bar{k}} \triangleq$ $\sqrt{\frac{\lambda_{k}}{\lambda_{\bar{k}}}} \frac{|\bar{u}(k)|^{2}}{\|\overline{\mathbf{u}}\|_{\Lambda}^{2}}+\sqrt{\frac{\lambda_{\bar{k}}}{\lambda_{k}}} \frac{|\bar{u}(\bar{k})|^{2}}{\|\overline{\mathbf{u}}\|_{\Lambda}^{2}}, q_{k} \triangleq \frac{|\bar{u}(k)|^{2}}{\left(\|\overline{\mathbf{u}}\|_{\Lambda}^{2}\right)^{2}}, z_{k} \triangleq \frac{|\bar{u}(k)|^{2}+1}{\|\overline{\mathbf{u}}\|_{\Lambda}^{2}}$, and, $r \triangleq \frac{1}{\|\overline{\mathbf{u}}\|_{\Lambda}^{2}}$. As summarized in Table I, the entries of the moment matrices (7) can be written in terms of these scalar random variables. It is easy to see that these random variables are either some ratio of quadratic forms or a linear combination of a ratio of two quadratic forms, a fact that we exploit to evaluate the required moments of these variables. Due to space limitation, we only provide the final expressions here and delegate the derivation to the companion report [22].

For $\overline{\mathbf{A}}_{\mathrm{N}}(k, k)$, we need to evaluate $\mathbb{E}\left[s_{k}\right]$, which is given as

$$
\mathbb{E}\left[s_{k}\right]=\frac{p_{k}}{\lambda_{k}}+\sum_{i=1, i \neq k}^{M} p_{i} \frac{\ln \left(\frac{\lambda_{i}}{\lambda_{k}}\right)}{\left(\lambda_{i}-\lambda_{k}\right)}
$$

where

$$
p_{i} \triangleq \frac{\lambda_{i}^{M-1}}{\prod_{j=1, j \neq i}^{M}\left(\lambda_{i}-\lambda_{j}\right)}, \forall i
$$

For $\overline{\mathbf{B}}_{\mathrm{N}}(k, \bar{k})$, we need to evaluate $\mathbb{E}\left[s_{k}^{2}\right]$, which is given as

$$
\mathbb{E}\left[s_{k}^{2}\right]=\frac{p_{k}}{\lambda_{k}^{2}}+\sum_{i=1, i \neq k}^{M} 2 p_{i} \frac{\left(\lambda_{i}-\lambda_{k}\right)-\lambda_{k} \ln \left(\frac{\lambda_{i}}{\lambda_{k}}\right)}{\lambda_{k}\left(\lambda_{i}-\lambda_{k}\right)^{2}} .
$$

TABLE I: Relation of $\overline{\mathbf{A}}_{\mathrm{N}}, \overline{\mathbf{B}}_{\mathrm{N}}$, and $\overline{\mathbf{C}}_{\mathrm{N}}$ to the moments of $s_{k}, s_{k \bar{k}}, q_{k}, z_{k}$ and, $r$ (see [22] for details).

\begin{tabular}{|c|c|}
\hline $\begin{array}{c}\text { Moment } \\
\text { matrix } \\
\text { entries }\end{array}$ & $\begin{array}{c}\text { Relation to the quadratic form } \\
\text { random variables }\end{array}$ \\
\hline$\overline{\mathbf{A}}_{\mathrm{N}}(k, k)$ & $2 \mathbb{E}\left[s_{k}\right]$ \\
\hline$\overline{\mathbf{A}}_{\mathrm{N}}(k, \bar{k})$ & 0 \\
\hline$\overline{\mathbf{B}}_{\mathrm{N}}(k, \bar{k})$ & $\mathbb{E}\left[s_{k}^{2}\right]$ \\
\hline$\overline{\mathbf{B}}_{\mathrm{N}}(k, \bar{k})$ & $\mathbb{E}\left[s_{k} s_{\bar{k}}\right]=\frac{1}{2}\left(\mathbb{E}\left[s_{k \bar{k}}^{2}\right]-\frac{\lambda_{k}}{\lambda_{\bar{k}}} \mathbb{E}\left[s_{k}^{2}\right]-\frac{\lambda_{\bar{k}}}{\lambda_{k}} \mathbb{E}\left[s_{\bar{k}}^{2}\right]\right)$ \\
\hline$\overline{\mathbf{C}}_{\mathrm{N}}(k, k)$ & $\mathbb{E}\left[q_{k}\right]=\frac{1}{2} \mathbb{E}\left[z_{k}^{2}\right]-\frac{1}{2} \mathbb{E}\left[s_{k}^{2}\right]-\frac{1}{2} \mathbb{E}\left[r^{2}\right]$ \\
\hline$\overline{\mathbf{C}}_{\mathrm{N}}(k, \bar{k})$ & 0 \\
\hline
\end{tabular}

For $\overline{\mathbf{B}}_{\mathrm{N}}(k, \bar{k})$, we need $\mathbb{E}\left[s_{k}^{2}\right]$ (given in (10)), $\mathbb{E}\left[s_{\bar{k}}^{2}\right]$, and $\mathbb{E}\left[s_{k \bar{k}}^{2}\right]$ (evaluated in (11) on the bottom of next page). Next $\overline{\mathbf{C}}_{\mathrm{N}}(k, k)$ requires $\mathbb{E}\left[s_{k}^{2}\right]$ (given in (10)), $\mathbb{E}\left[z_{k}^{2}\right]$ and $\mathbb{E}\left[r^{2}\right]$. For $\mathbb{E}\left[z_{k}^{2}\right]$, let us define the Beta function $B(x, y)$

$$
B(x, y) \triangleq \int_{0}^{1} t^{x-1}(1-t)^{y-1} d t, \Re(x)>0, \Re(y)>0,
$$

and the Hypergeometric Function ${ }_{2} F_{1}(\alpha, \beta ; \gamma ; x)$ given in (13) at the bottom of the next page. Next, let $\eta_{i k}=1-\frac{\lambda_{i}}{\lambda_{k}}$, and denote the determinant of the matrix $\boldsymbol{\Lambda}$ as $|\boldsymbol{\Lambda}|$ to get $\mathbb{E}\left[z_{k}^{2}\right]$ as given in (14) at the bottom of next page. Finally, $\mathbb{E}\left[r^{2}\right]$ can be expressed as

$$
\mathbb{E}\left[r^{2}\right]=\sum_{i=1}^{M} \frac{\lambda_{i}^{M}}{|\boldsymbol{\Lambda}| \prod_{j=1, j \neq i}^{M}\left(\frac{\lambda_{i}}{\lambda_{j}}-1\right)} .
$$

\section{SENSITIVITY COMPARISON OF THE MOMENT MATRICES TO EIGENVALUES}

We now numerically compare the moment matrices of LMS (4) and the moment matrices of NLMS derived in Section II. Specifically, the objective of the numerical study is to show that as $\boldsymbol{\lambda}$ changes, the moment matrices of the LMS vary more than the moment matrices of the NLMS. For numerical illustration, we choose the filter length to be $M=5$. Further, let $\mathcal{U}[0,1]$ denote the standard Uniform distribution. We obtain 1000 independent realizations of $\boldsymbol{\lambda}$, where $\lambda_{k} \sim \mathcal{U}[0,1], \forall k$, and compare the empirical CDFs, $F(x)$, of the entries of moment matrices, $x$.

The CDFs for $\mathbf{A}_{\mathrm{L}}(k, k)$ and $\mathbf{A}_{\mathrm{N}}(k, k)$ are shown in Fig. 1a. For the problem at hand, whenever the eigenvalues $\lambda_{k}$ have the same distribution for all $k, \mathbf{A}_{\mathrm{L}}(k, k)$ and $\mathbf{A}_{\mathrm{N}}(k, k)$ will also have the same distribution for all $k$ (also true for $\mathbf{B}$ and C), so we draw the CDF of a randomly selected $k$ th entry. As expected from the closed-form expression of $\mathbf{A}_{\mathrm{L}}(k, k)$ in (4), the $\mathrm{CDF}$ is a straight line. The $\mathrm{CDF}$ of $\mathbf{A}_{\mathrm{N}}(k, k)$, however, is steeper and more concentrated. This implies that as eigenvalues change, the entries $\mathbf{A}_{\mathrm{N}}(k, k)$ show less variation than $\mathbf{A}_{\mathrm{L}}(k, k)$. For $\mathbf{B}_{\mathrm{L}}$ and $\mathbf{B}_{\mathrm{N}}$, we study the diagonal and nondiagonal entries separately in Fig. 1b. The CDF of $\mathbf{B}_{\mathrm{N}}(k, k)$ is steeper than $\mathbf{B}_{\mathrm{L}}(k, k)$, and the $\mathrm{CDF}$ for $\mathbf{B}_{\mathrm{N}}(k, \bar{k})$ is steeper than $\mathbf{B}_{\mathrm{L}}(k, \bar{k})$. Finally, in Fig. 1c, we show the CDF curves of $\mathbf{C}_{\mathrm{L}}(k, k)$ and $\mathbf{C}_{\mathrm{N}}(k, k)$, and see similar behavior about the steepness of $\mathbf{C}_{\mathrm{N}}(k, k)$ compared to $\mathbf{C}_{\mathrm{L}}(k, k)$. Together, all the 
CDF curves in Fig. 1 show that as $\boldsymbol{\lambda}$ changes, the moment matrices of NLMS vary less than the moment matrices of LMS.

\section{SENSITIVITY COMPARISON OF THE MOMENT MATRICES TO EIGENVALUE-SPREAD}

The objective of this section is to show that the moment matrices of the LMS are more sensitive to the eigenvaluespread than those of the NLMS. To this end, assume without loss of generality that the eigenvalues are ordered as $0 \leq \lambda_{1} \leq$ $\lambda_{2} \leq \cdots \leq \lambda_{M}$, and let $\rho=\frac{\lambda_{M}}{\lambda_{1}}$ denote the eigenvalue-spread. To quantify the variation in the entries of moment matrices, we use range, i.e., the difference between the largest entry and the smallest entry of a moment matrix. As $\rho$ increases, the range of the moment matrices will increase for both LMS and NLMS. We, however, expect the range increase of LMS moment matrices to be higher than their NLMS counterparts. In other words, the difference in the ranges of LMS and NLMS moment matrices should increase with $\rho$. We outline the desired result via the following theorem

Theorem 1. Let $\max (\mathbf{X})$ and $\min (\mathbf{X})$ denote the largest and smallest values in the matrix $\mathbf{X}$ respectively, and let $\mathrm{R}(\mathbf{X})=$ $\max (\mathbf{X})-\min (\mathbf{X})$ be the range, then the difference

$$
\Delta\left(\mathbf{A}_{\mathrm{L}}, \mathbf{A}_{\mathrm{N}}\right)=\mathrm{R}\left(\mathbf{A}_{\mathrm{L}}\right)-\mathrm{R}\left(\mathbf{A}_{\mathrm{N}}\right)
$$

is an increasing function of $\rho$. Let $\Delta\left(\mathbf{B}_{\mathrm{L}}, \mathbf{B}_{\mathrm{N}}\right)$ and $\Delta\left(\mathbf{C}_{\mathrm{L}}, \mathbf{C}_{\mathrm{N}}\right)$ be analogously defined, then these functions are also increasing functions of $\rho$.

Proof. For the proof, we use the derivative test, i.e., we seek to show that $\frac{\partial}{\partial \rho} \Delta\left(\mathbf{A}_{\mathrm{L}}, \mathbf{A}_{\mathrm{N}}\right)>0, \frac{\partial}{\partial \rho} \Delta\left(\mathbf{B}_{\mathrm{L}}, \mathbf{B}_{\mathrm{N}}\right)>0$, and $\frac{\partial}{\partial \rho} \Delta\left(\mathbf{C}_{\mathrm{L}}, \mathbf{C}_{\mathrm{N}}\right)>0$.

Note that $\rho$ does not change when the vector $\boldsymbol{\lambda}$ is scaled i.e., $\kappa \lambda$ for $\kappa>0$. As the relative performance of the LMS and NLMS changes with $\rho$, scaling the eigenvalue vector $\boldsymbol{\lambda}$ is inconsequential in the relative performance analysis. Care, however, must be taken in choosing the step-size of the algorithms. The step-size of the NLMS does not need to be changed with the scaling, whereas, the step-size of the LMS should account for the scaling. With this understanding, we can choose $\kappa$ for analytic convenience. Specifically, we choose $\kappa$ to have $\lambda_{1}=1$, and with that $\rho=\lambda_{M}$. Thus, if we denote the partial derivative of a variable $x$ with respect to $\lambda_{M}$ as $x^{\prime} \triangleq \frac{\partial x}{\partial \lambda_{M}}$, we need to show that $\Delta^{\prime}\left(\mathbf{A}_{\mathrm{L}}, \mathbf{A}_{\mathrm{N}}\right)>0$, $\Delta^{\prime}\left(\mathbf{B}_{\mathrm{L}}, \mathbf{B}_{\mathrm{N}}\right)>0$, and $\Delta^{\prime}\left(\mathbf{C}_{\mathrm{L}}, \mathbf{C}_{\mathrm{N}}\right)>0$.

We start by showing that $\Delta^{\prime}\left(\mathbf{A}_{\mathrm{L}}, \mathbf{A}_{\mathrm{N}}\right)>0$. To obtain $\mathrm{R}\left(\mathbf{A}_{\mathrm{L}}\right)$, it is easy to see that $\max \left(\mathbf{A}_{\mathrm{L}}\right)=2 \lambda_{M}$ and $\min \left(\mathbf{A}_{\mathrm{L}}\right)=0$. For $\mathbf{A}_{\mathrm{N}}$, from (4) and (5) note that the entries in $\mathbf{A}_{\mathrm{N}}$ are the expectation of the normalized versions of the entries of $\mathbf{A}_{\mathrm{L}}$, where the same normalization is used for all entries. Therefore, the normalization cannot change the relative order of the entries. Thus, $\max \left(\mathbf{A}_{\mathrm{N}}\right)=2 \lambda_{M} \mathbb{E}\left[s_{M}\right]$ and $\min \left(\mathbf{A}_{\mathrm{N}}\right)=0$. Therefore, we have

$$
\Delta\left(\mathbf{A}_{\mathrm{L}}, \mathbf{A}_{\mathrm{N}}\right)=2 \lambda_{M}-2 \lambda_{M} \mathbb{E}\left[s_{M}\right]
$$

At this stage, we make a few observations. First, while evaluating the derivative of $\mathbb{E}\left[s_{M}\right]$, the role of expectation and derivative can be interchanged, i.e., $\left(\mathbb{E}\left[s_{M}\right]\right)^{\prime}=\mathbb{E}\left[s_{M}^{\prime}\right]$, and $s_{M}^{\prime}$ is given as

$$
s_{M}^{\prime}=\frac{\partial}{\partial \lambda_{M}} \frac{|\bar{u}(M)|^{2}}{\|\overline{\mathbf{u}}\|_{\Lambda}^{2}}=-\left(\frac{|\bar{u}(M)|^{2}}{\|\overline{\mathbf{u}}\|_{\Lambda}^{2}}\right)^{2}=-s_{M}^{2} .
$$

Second, $s_{M}$ has support $\left[0, \frac{1}{\lambda_{M}}\right]$, therefore,

$$
0<\mathbb{E}\left[s_{M}\right]<\frac{1}{\lambda_{M}}, \text { and } 0<\mathbb{E}\left[s_{M}^{2}\right]<\frac{1}{\lambda_{M}^{2}} .
$$

$$
\begin{aligned}
& \mathbb{E}\left[s_{k \bar{k}}^{2}\right]=\frac{p_{k}}{\lambda_{k} \lambda_{\bar{k}}}-\frac{2(-1)^{M-1}\left(\frac{\lambda_{k}}{\lambda_{\bar{k}}}\right)^{(M-2) / 2}}{\sqrt{\lambda_{k} \lambda_{\bar{k}}}}\left\{\sum_{i=1, i \neq k, \bar{k}}^{M} \frac{p_{i}\left(1+\ln \left(\frac{\lambda_{k}}{\lambda_{i}}\right)\right)}{\left(\lambda_{i}-\lambda_{k}\right)^{M-2}\left[1-\frac{\left(\frac{\lambda_{\bar{k}}}{\lambda_{k}}\right)^{2}\left(\lambda_{i}-\lambda_{k}\right)}{\left(\lambda_{i}-\lambda_{\bar{k}}\right)}\right]}\right. \\
& \left.+\sum_{i=1, i \neq k, \bar{k}}^{M} \frac{p_{i}\left(1+\ln \left(\frac{\lambda_{\bar{k}}}{\lambda_{i}}\right)\right)}{\left(\lambda_{i}-\lambda_{\bar{k}}\right)^{M-2}\left[1-\frac{\left(\frac{\lambda_{k}}{\lambda_{\bar{k}}}\right)^{2}\left(\lambda_{i}-\lambda_{\bar{k}}\right)}{\left(\lambda_{i}-\lambda_{k}\right)}\right]}\right\} \text {. } \\
& { }_{2} F_{1}(\alpha, \beta ; \gamma ; x) \triangleq \frac{1}{B(\beta, \gamma-\beta)} \int_{0}^{1} x^{\beta-1}(1-x)^{\gamma-\beta-1}(1-x z)^{-\alpha} d x, \Re(\gamma)>\Re(\beta)>0 . \\
& \mathbb{E}\left[z_{k}^{2}\right]=\sum_{i=1, i \neq k}^{M} \frac{\ln \left(\lambda_{i}\right)}{|\boldsymbol{\Lambda}| \prod_{j=1, j \neq i, k}^{M}\left(\frac{1}{\lambda_{j}}-\frac{1}{\lambda_{i}}\right)}+\sum_{i=1, i \neq k}^{M} \frac{\lambda_{i}\left(1+{ }_{2} F_{1}\left(1,1 ; 2 ; \eta_{i k}\right)-{ }_{2} F_{1}\left(1,1 ; 3 ; \eta_{i k}\right)\right)}{\lambda_{k}|\boldsymbol{\Lambda}| \prod_{j=1, j \neq i, k}^{M}\left(\frac{1}{\lambda_{j}}-\frac{1}{\lambda_{i}}\right)} \\
& +\sum_{i=1, i \neq k}^{M} \frac{\lambda_{i}^{2}}{\lambda_{k}|\boldsymbol{\Lambda}| \prod_{j=1, j \neq i, k}^{M}\left(\frac{1}{\lambda_{j}}-\frac{1}{\lambda_{i}}\right)}\left(\frac{1}{3}{ }_{2} F_{1}\left(1,2 ; 4 ; \eta_{i k}\right)-{ }_{2} F_{1}\left(1,2 ; 3 ; \eta_{i k}\right)\right) \text {. }
\end{aligned}
$$




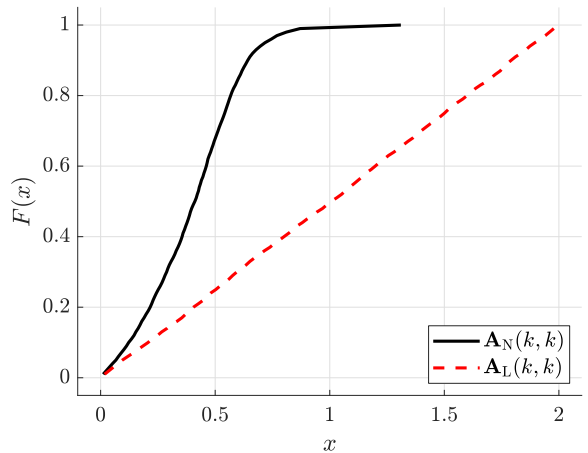

(a) The CDFs for entries of $\mathbf{A}_{L}$ and $\mathbf{A}_{N}$.

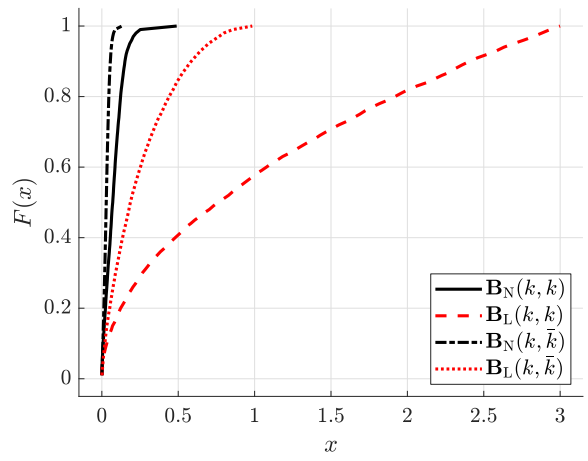

(b) The CDFs for entries of $\mathbf{B}_{\mathrm{L}}$ and $\mathbf{B}_{\mathrm{N}}$.

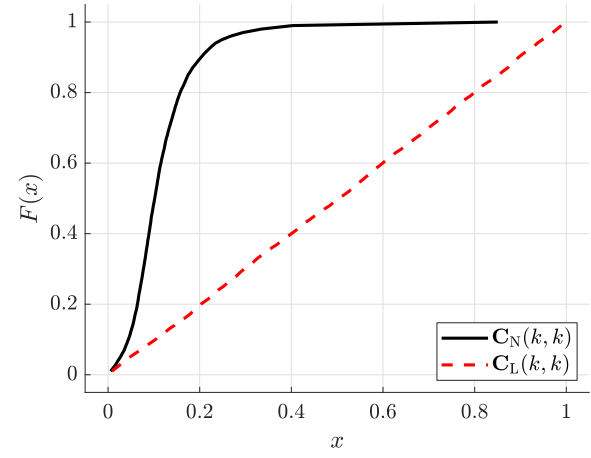

(c) The CDFs for entries of $\mathbf{C}_{\mathrm{L}}$ and $\mathbf{C}_{\mathrm{N}}$.

Fig. 1: CDF comparison of the moment matrices of LMS and NLMS for a filter of length $M=5$ and uniformly distributed eigenvalues.

With the aforementioned observations, we have

$$
\begin{aligned}
\Delta^{\prime}\left(\mathbf{A}_{\mathrm{L}}, \mathbf{A}_{\mathrm{N}}\right) & \stackrel{(a)}{=} 2-2 \mathbb{E}\left[s_{M}\right]+2 \lambda_{M} \mathbb{E}\left[s_{M}^{2}\right] \\
& \stackrel{(b)}{>} 2-\frac{2}{\lambda_{M}} \stackrel{(c)}{\geq} 0,
\end{aligned}
$$

where $(a)$ follows from (17), (18), and simple algebra. Next, (b) follows from (19), lower bounding the positive terms, and upper bounding the negative terms. Finally, $(c)$ is true as $\lambda_{M} \geq$ 1 , since $1=\lambda_{1} \leq \lambda_{2} \leq \cdots \leq \lambda_{M}$.

Next, we show that $\Delta^{\prime}\left(\mathbf{B}_{\mathrm{L}}, \mathbf{B}_{\mathrm{N}}\right)>0$. For $\mathbf{B}_{\mathrm{L}}, \max \left(\mathbf{B}_{\mathrm{L}}\right)=$ $3 \lambda_{M}^{2}$, where $\min \left(\mathbf{B}_{\mathrm{L}}\right)$ is either $3 \lambda_{1}^{2}$ or $\lambda_{1} \lambda_{2}$, depending on the numerical value of $\lambda_{1}$ and $\lambda_{2}$. Thus, we have $\min \left(\mathbf{B}_{\mathrm{L}}\right)=\min \left(3 \lambda_{1}^{2}, \lambda_{1} \lambda_{2}\right)$. As normalization in (5) does not impact ordering, $\max \left(\mathbf{B}_{\mathrm{N}}\right)=\lambda_{M}^{2} \mathbb{E}\left[s_{M}^{2}\right]$, and $\min \left(\mathbf{B}_{\mathrm{N}}\right)=$ $\min \left(\lambda_{1}^{2} \mathbb{E}\left[s_{1}^{2}\right], \lambda_{1} \lambda_{2} \mathbb{E}\left[s_{1} s_{2}\right]\right)$. We divide the proof in two parts depending on the exact value of $\min \left(\mathbf{B}_{\mathrm{L}}\right)$. First, we consider $\min \left(\mathbf{B}_{\mathrm{L}}\right)=3 \lambda_{1}^{2}$ and $\min \left(\mathbf{B}_{\mathrm{N}}\right)=\lambda_{1}^{2} \mathbb{E}\left[s_{1}^{2}\right]$, so that

$$
\Delta\left(\mathbf{B}_{\mathrm{L}}, \mathbf{B}_{\mathrm{N}}\right)=\left(3 \lambda_{M}^{2}-3 \lambda_{1}^{2}\right)-\left(\lambda_{M}^{2} \mathbb{E}\left[s_{M}^{2}\right]-\lambda_{1}^{2} \mathbb{E}\left[s_{1}^{2}\right]\right) .
$$

The required derivatives for the proof are

$$
\left(s_{M}^{2}\right)^{\prime}=2 s_{M} s_{M}^{\prime}=-2 s_{M}^{3},
$$

and

$$
\left(s_{1}^{2}\right)^{\prime}=2 s_{1} s_{1}^{\prime}=-2 s_{1}^{2} s_{M} .
$$

Also based on the support of variables $s_{M}^{2}, s_{M}^{3}$, and $s_{1} s_{M}$, we have the following bounds

$$
0<\mathbb{E}\left[s_{M}^{3}\right]<\frac{1}{\lambda_{M}^{3}}, \text { and } 0<\mathbb{E}\left[s_{1} s_{M}\right]<\frac{1}{\lambda_{1} \lambda_{M}} .
$$

From observations above, we have

$$
\begin{aligned}
& \Delta^{\prime}\left(\mathbf{B}_{\mathrm{L}}, \mathbf{B}_{\mathrm{N}}\right) \\
& \stackrel{(a)}{=} 6 \lambda_{M}-2 \lambda_{M} \mathbb{E}\left[s_{M}^{2}\right]+2 \lambda_{M}^{2} \mathbb{E}\left[s_{M}^{3}\right] \\
& \quad-\lambda_{1}^{2} \mathbb{E}\left[s_{1} s_{M}\right] \stackrel{(b)}{>} 6 \lambda_{M}-\frac{2}{\lambda_{M}} \stackrel{(c)}{>} 0,
\end{aligned}
$$

where (a) follows from (21)-(23). Next, (b) follows from (19), (24), by lower bounding the positive terms, and upper bounding the negative terms. Finally $(c)$ is true as long as $\lambda_{M} \geq \sqrt{\frac{1}{3}}$, which is always satisfied.

Now consider the case $\min \left(\mathbf{B}_{\mathrm{L}}\right)=\lambda_{1} \lambda_{2}$ and $\min \left(\mathbf{B}_{\mathrm{N}}\right)=$ $\lambda_{1} \lambda_{2} \mathbb{E}\left[s_{1} s_{2}\right]$. Here, we need the derivative

$$
\left(s_{1} s_{2}\right)^{\prime}=s_{1} s_{2}^{\prime}+s_{2} s_{1}^{\prime}=-s_{1} s_{2} s_{M}-s_{2} s_{1} s_{M}=-2 s_{1} s_{2} s_{M},
$$

and the bound $0<\mathbb{E}\left[s_{1} s_{2} s_{M}\right]<\frac{1}{\lambda_{1} \lambda_{2} \lambda_{M}}$. With these, we proceed in a manner identical to (25) to get

$$
\Delta^{\prime}\left(\mathbf{B}_{\mathrm{L}}, \mathbf{B}_{\mathrm{N}}\right)>6 \lambda_{M}-\frac{4}{\lambda_{M}} \stackrel{(a)}{>} 0
$$

where $(a)$ holds as long as $\lambda_{M} \geq \sqrt{\frac{2}{3}}$, which is always satisfied.

Finally, to show that $\Delta^{\prime}\left(\mathbf{C}_{\mathrm{L}}, \mathbf{C}_{\mathrm{N}}\right)>0$, note that $\max \left(\mathbf{C}_{\mathrm{L}}\right)=\lambda_{M}, \min \left(\mathbf{C}_{\mathrm{L}}\right)=0, \max \left(\mathbf{C}_{\mathrm{N}}\right)=\lambda_{M} \mathbb{E}\left[k_{M}\right]$, and $\min \left(\mathbf{C}_{\mathrm{N}}\right)=0$. So we have $\Delta\left(\mathbf{C}_{\mathrm{L}}, \mathbf{C}_{\mathrm{N}}\right)=\lambda_{M}-2 \lambda_{M} \mathbb{E}\left[k_{M}\right]$.

Using the derivative $k_{M}^{\prime}=-2 k_{M} s_{M}$, the bounds $0<$ $\mathbb{E}\left[k_{M}\right]<\frac{1}{\lambda_{M}^{2}}, 0<\mathbb{E}\left[k_{M} s_{M}\right]<\frac{1}{\lambda_{M}^{3}}$, and proceeding in a manner similar to (20) and (25), we get

$$
\Delta^{\prime}\left(\mathbf{C}_{\mathrm{L}}, \mathbf{C}_{\mathrm{N}}\right)>1-\frac{1}{\lambda_{M}^{2}} \stackrel{(a)}{\geq} 0
$$

where $(a)$ holds as $\lambda_{M} \geq 1$. Together, (20), (25) and (28) complete the proof.

\section{CONCLUSION AND FUTURE WORK}

This paper attempted to explain the better convergence speed of the NLMS compared to the LMS for correlated input signals. We utilized the fact that the mean-square behavior of the two algorithms follows the same variance relation parameterized by different moment matrices. We derived the closed-form expressions of these moment matrices. We then showed by simulations that those of the NLMS are less sensitive to variations in eigenvalues than those of the LMS. We also showed analytically that the moment matrices of the NLMS are less sensitive to eigenvalue spread than the LMS.

This work provided a framework for analyzing the impact of eigenvalue-spread on mean-square performance and in the future can be used for comparative analysis of other algorithms. 


\section{REFERENCES}

[1] S. Haykin, Adaptive Filter Theory. Englewood Cliffs, NJ: PrenticeHall, 1996.

[2] A. H. Sayed, Adaptive Filters. John Wiley \& Sons, 2011.

[3] B. Widrow and S. D. Stearns, Adaptive Signal Processing. Englewood Cliffs, NJ: Prentice-Hall, 1985.

[4] B. Widrow, J. McCool, M. G. Larimore, and C. R. Johnson, "Stationary and nonstationary learning characteristics of the LMS adaptive filter," Proc. IEEE, vol. 64, no. 8, pp. 1151-1162, Aug. 1976.

[5] J.-I. Nagumo and A. Noda, "A learning method for system identification," IEEE Trans. Autom. Control, vol. 12, no. 3, pp. 282-287, 1967.

[6] A. Feuer and E. Weinstein, "Convergence analysis of LMS filters with uncorrelated Gaussian data," IEEE Trans. Acoust., Speech, Signal Process., vol. 33, no. 1, pp. 222-230, 1985.

[7] S. C. Douglas and W. Pan, "Exact expectation analysis of the LMS adaptive filter," IEEE Trans. Signal Process., vol. 43, no. 12, pp. $2863-$ 2871, 1995.

[8] G. Barrault, M. H. Costa, J. C. Bermudez, and A. Lenzi, "A new analytical model for the NLMS algorithm," in Proc. IEEE Int. Conf. Acoust., Speech Signal Process. (ICASSP). IEEE, 2005, pp. 41-44.

[9] E. M. Lobato, O. J. Tobias, and R. Seara, "Stochastic model for the NLMS algorithm with correlated Gaussian data," in Proc. IEEE Int. Conf. Acoust., Speech Signal Process. (ICASSP), vol. 3. IEEE, 2006, pp. $760-763$.

[10] M. H. Costa and J. C. Bermudez, "An improved model for the normalized LMS algorithm with Gaussian inputs and large number of coefficients," in Proc. IEEE Int. Conf. Acoust., Speech Signal Process. (ICASSP), vol. 2. IEEE, 2002, pp. 1385-1388.

[11] A. Ali, M. Moinuddin, and T. Y. Al-Naffouri, "The NLMS Is SteadyState Schur-Convex," IEEE Signal Processing Letters, vol. 28, pp. 389393,2021
[12] M. Tarrab and A. Feuer, "Convergence and performance analysis of the normalized LMS algorithm with uncorrelated Gaussian data," IEEE Trans. Inf. Theory, vol. 34, no. 4, pp. 680-691, 1988.

[13] T. Y. Al-Naffouri, M. Moinuddin, and M. S. Sohail, "Mean weight behavior of the NLMS algorithm for correlated Gaussian inputs," IEEE Signal Process. Lett., vol. 18, no. 1, pp. 7-10, 2010.

[14] N. J. Bershad, E. Eweda, and J. C. Bermudez, "Stochastic analysis of the LMS and NLMS algorithms for cyclostationary white Gaussian inputs," IEEE Trans. Signal Process., vol. 62, no. 9, pp. 2238-2249, 2014.

[15] E. Eweda, N. J. Bershad, and J. C. M. Bermudez, "Stochastic analysis of the LMS and NLMS algorithms for cyclostationary white Gaussian and non-Gaussian inputs," IEEE Trans. Signal Process., vol. 66, no. 18 pp. $4753-4765,2018$.

[16] D. T. Slock, "On the convergence behavior of the LMS and the normalized LMS algorithms," IEEE Trans. Signal Process., vol. 41, no. 9, pp. 2811-2825, 1993.

[17] S. Zhang, J. Zhang, and H. C. So, "Mean square deviation analysis of LMS and NLMS algorithms with white reference inputs," Signal Process., vol. 131, pp. 20-26, 2017.

[18] M. Rupp and R. Frenzel, "Analysis of LMS and NLMS algorithms with delayed coefficient update under the presence of spherically invariant processes," IEEE Trans. Signal Process., vol. 42, no. 3, pp. 668-672, 1994.

[19] T. Y. Al-Naffouri and A. H. Sayed, "Transient analysis of datanormalized adaptive filters," IEEE Trans. Signal Process., vol. 51, no. 3, pp. 639-652, 2003.

[20] T. Y. Al-Naffouri and M. Moinuddin, "Exact Performance Analysis of the $\epsilon$-NLMS Algorithm for Colored Circular Gaussian Inputs," IEEE Trans. Signal Process., vol. 58, no. 10, pp. 5080-5090, 2010.

[21] S. C. Douglas and T.-Y. Meng, "Normalized data nonlinearities for LMS adaptation," IEEE Trans. Signal Process., vol. 42, no. 6, pp. 1352-1365, 1994.

[22] T. Y. Al-Naffouri, M. Moinuddin, and A. Ali, "Mean-square Analysis of the NLMS Algorithm," arXiv:2108.03721, 2021. 